J. Satoh

Nagoya Math. J.

Vol. 127 (1992), 129-143

\title{
A CONSTRUCTION OF $q$-ANALOGUE OF DEDEKIND SUMS
}

\author{
JUNYA SATOH
}

\section{Introduction}

If one looks back the classical proof (cf. Carlitz [4]) of the reciprocity law for Dedekind sums in order to construct $q$-analogue of Dedekind sums which also have the reciprocity law, one can soon see that the following elementary equation is essential in the proof:

$$
\frac{1-u}{e^{t}-u} \frac{1-v}{e^{t}-v}=\frac{1-v}{u-v} \frac{1-u}{e^{t}-u}+\frac{1-u}{v-u} \frac{1-v}{e^{t}-v},
$$

for any distinct complex numbers $u$ and $v$, where $\frac{1-u}{e^{t}-u}$ means the generating function of Euler numbers associated to $u$. So we must extend the above equation to the generating function of $q$-Euler numbers for our purpose. As a result, we obtain a very suggestive equation (see Lamma 5 ) under the conditions $|u|>1$ and $|v|>1$ :

$$
F_{u ; q}(t) * F_{v ; q}(t)=\frac{1-v}{u-v} F_{u ; q}(t)+\frac{1-u}{v-u} F_{v ; q}(t),
$$

where $F_{u ; q}(t)$ means the generating function of $q$-Euler numbers associated to $u$ and the left hand side of (2) is determined by Lemma 4 . The above equation is correspond to the decomposition into partial fractions of $\frac{1-u}{e^{t}-u} \frac{1-v}{e^{t}-v}:$ (1). We take a deep interest in the invariance of the form. By the generalization of the theory, we give a new method of construction of $q$-analogue of formal power series. In the following, we explain about the essence of our theory. In [2], Carlitz defined $q$-Bernoulli numbers for a complex number $q$ as follows:

$$
\beta_{0}(q)=1, \quad q(q \beta(q)+1)^{n}-\beta_{n}(q)= \begin{cases}1 & \text { for } n=1 \\ 0 & \text { for } n>1\end{cases}
$$

Received November 6, 1990. 
with the usulal convention about replacing $\beta^{m}(q)$ by $\beta_{m}(q)$. However it is almost impossible to extend the above recurrence definition to arbitrary sequence, harmonizing with $q$-Bernoulli numbers. So we need to consider another construction of $q$-Bernoulli numbers. For that reason, in the next place, we explain another construction of $q$-Bernoulli numbers from the position that the generating function of $q$-Bernoulli numbers can be viewed as a solution of $q$-difference equation, following the author's previous paper [6]. Let $G_{q}(t)$ be the generating function of $q$-Bernoulli numbers, i.e.,

$$
G_{q}(t)=\sum_{n=0}^{\infty} \beta_{n}(q) \frac{t^{n}}{n !}
$$

Then $G_{q}(t)$ is determined as a unique solution of the following $q$-difference equation $[6,(2)]$ :

$$
G_{q}(t)=q e^{t} G_{q}(q t)-t-q+1 .
$$

If $|q|<1$, then the solution of the above $q$-difference equation is expressed as follows $[6$, Lemma 1$]$ :

$$
G_{q}(t)=-\sum_{n=0}^{\infty} q^{n} e^{[n] t}\left(q^{n} t+q-1\right)
$$

where $[x]=[x ; q]$ means $\frac{q^{x}-1}{q-1}$ for any complex number $x$.

Now the generating function of classical (i.e., in the case of $q=1$ ) Bernoulli numbers is formally expressed as follows:

$$
G_{1}(t)=\frac{t}{e^{t}-1}=-\sum_{n=0}^{\infty} e^{n t} t,
$$

so we can easily imagine that

$$
-\sum_{n=0}^{\infty} q^{n} e^{[n] t} t
$$

may be suitable for $q$-analogue of $G_{1}(t)$. In fact, if we use an operator $\varphi_{q}$ such that

$$
\varphi_{q}=1_{\mathrm{id}}+(q-1) \frac{d}{d t},
$$

then $G_{q}(t)$ is expressed as follows:

$$
G_{q}(t)=\varphi_{q}\left(-\sum_{n=0}^{\infty} q^{n} e^{[n] t} t\right) .
$$

The purpose of this paper is that we give a new method of construction of $q$-analogue using the above operator $\varphi_{q}$. As the first step, we extend the map: $G_{1}(t) \mapsto G_{q}(t)$ to general power series using $\varphi_{q}$ and $*$. Next we show that the map is a homomorphism. So we can deduce unknown $q$-formulae from known 
classical formulae. For exmple, we can deduce (2) from (1).

In the following part, we describe the construction of $q$-analogue of Dedekind sums in order to explain the background of our theory. In the second part, we bring about our purpose and review the argument of the first part from a new angle of vision.

\section{Construction of $q$-Dedekind sums}

\section{1-1. Notation and properties of $\alpha_{n}(x ; q)$}

Throughout this paper, we assume that $q$ and $u$ are complex numbers such that $|q|<1$ and $|u|>1$. Following Carlitz [5], we define $q$-Euler numbers associated to $u$ by

$$
H_{0}(q ; u)=1, \quad(q H(q ; u)+1)^{n}-u H_{n}(q ; u)=0 \text { for } n>0,
$$

and $q$-Bernoulli polynomials and $q$-Euler polynomials by

$$
\beta_{n}(x ; q)=\sum_{m=0}^{n}\left(\begin{array}{l}
n \\
m
\end{array}\right) q^{m x} \beta_{m}(q)[x]^{n-m}
$$

and

$$
H_{n}(x, u ; q)=\sum_{m=0}^{n}\left(\begin{array}{l}
n \\
m
\end{array}\right) q^{m x} H_{m}(u ; q)[x]^{n-m},
$$

respectively. In particular, we define the following:

Definition 1. For each non-negative integer $n$, we define

$$
\alpha_{n}(x ; q)=\frac{1}{q-1} H_{n}\left(x ; q^{-1} ; q\right) \text { and } a_{n}(q)=a_{n}(0 ; q) .
$$

These polynomials have the almost same properties with classical Bernoulli polynomials $B_{n}(x)$ and play an important role in this paper in spite of the divergency of $\lim _{q \rightarrow 1} \alpha_{n}(x ; q)$. In fact, (8) implies as a Laurent series

$$
\lim _{q \rightarrow 1} A_{q}(t)=\frac{G_{1}(t)}{t}
$$

where $A_{q}(t)$ and $G_{q}(t)$ mean the generating functions with indeterminate $t$ of $\alpha_{n}(q)$ and $\beta_{n}(q)$, respectively.

Now we study properties of $\alpha_{n}(x ; q)$ for $\geq 0$, which will be used to define our $q$-Dedekind sums $s_{n ; q}(h, k)$.

LEMMA 1. For any non-negative integer $n$ and positive integer $k$, we have 


$$
[k]^{n} \sum_{a=0}^{k-1}(q \zeta)^{a} \alpha_{n}\left(\frac{a}{k} ; q^{k}\right)=\frac{1}{q \zeta-1} H_{n}\left((q \zeta)^{-1} ; q\right),
$$

where $\zeta$ is a $k$-th root of unity.

Proof. By the definition of $\alpha_{n}(x ; q)$, the left hand side of $(4)$ is equal to

$$
[k]^{n} \sum_{a=0}^{k-1} \frac{(q \zeta)^{a}}{(q \zeta)^{k}-1} H_{n}\left(\frac{a}{k},(q \zeta)^{-k} ; q^{k}\right) .
$$

This is equal to the right hand side of (4) by [6, Lemma 2], which will be again proved in the last of this paper in consequence of our theory: (16).

Next we define $q$-analogue of Bernoulli (or Euler) functions as follows :

Definition 2. For each non-negative integer $n$, we define

$$
\bar{\alpha}_{n}(x ; q)=\alpha_{n}(\{x\} ; q) q^{\{x\}},
$$

where $\{x\}$ means the decimal part of $x$.

Using this notation, Lemma 1 is expressed as follows:

$$
[k]^{n} \sum_{a \bmod k} \zeta^{a} \bar{\alpha}_{n}\left(\frac{a}{k} ; q^{k}\right)=\frac{1}{q \zeta-1} H_{n}\left((q \zeta)^{-1} ; q\right) .
$$

By solving the above equality with respect to $\bar{\alpha}_{n}\left(\frac{a}{k} ; q^{k}\right)$, we obtain

LEMMA 2. For any non-negative integer $n$ and positive integer $k$, we have

$$
\bar{\alpha}_{n}\left(\frac{a}{k} ; q^{k}\right)=\frac{1}{[k]^{n} k} \sum_{\zeta^{k}=1} \frac{\zeta^{-a}}{q \zeta^{-1}-1} H_{n}\left((q \zeta)^{-1} ; q\right) .
$$

Proof. Since $\bar{\alpha}_{n}\left(\frac{a}{k} ; q^{k}\right)$ is a periodic function with period $k$ with respect to $a \in \mathbf{Z}$, they are represented as finite Fourier series:

$$
\bar{\alpha}_{n}\left(\frac{a}{k} ; q^{k}\right)=\sum_{s=0}^{k-1} C_{s} \xi^{-a s},
$$

where $\xi$ is a primitive $k$-th root of unity. Hence we obtain the following by (5)

$$
C_{s}=\frac{1}{k} \sum_{a=0}^{k-1} \xi^{a s} \bar{\alpha}_{n}\left(\frac{a}{k} ; q^{k}\right)=\frac{1}{[k]^{n} k} \frac{1}{q \xi^{s}-1} H_{n}\left(\left(q \xi^{s}\right)^{-1} ; q\right) .
$$

This completes the proof of Lemma 2.

Note that by $(3)$ and $[4,(6.4)]$ we have

$$
\lim _{q \rightarrow 1}\left\{\bar{\alpha}_{n}\left(\frac{a}{k} ; q^{k}\right)-\frac{\alpha_{n}}{[k]^{n} k}\right\}=\frac{1}{n+1}\left\{\bar{B}_{n+1}\left(\frac{a}{k}\right)-\frac{B_{n+1}}{k^{n+1}}\right\},
$$

where $\bar{B}_{n}(x)$ mean the $n$-th Bernoulli functions : $\bar{B}_{n}(x)=B_{n}(\{x\})$. 
Now we define $q$-analogue of Dedekind sums using $\bar{\alpha}_{n}(x ; q)$ as follows:

Definition 3. For each non-negative integer $n$ and for any positive integers $h$ and $k$, we define

$$
s_{n ; q}(h, k)=\sum_{a \bmod k} \bar{B}_{1}\left(\frac{a}{k}\right) \bar{\alpha}_{n}\left(\frac{a h}{k} ; q^{k}\right) .
$$

Then we obtain the following result which is an immediate consequence of Lemma 2:

Lemma 3. For any non-negative integer $n$ and positive integers $h$ and $k$ such that $(h, k)=1$, we have

$$
s_{n ; q}(h, k)=\frac{\alpha_{n}(q) B_{1}}{[k]^{n} k}+\frac{1}{[k]^{n} k} \sum_{\substack{\zeta^{k}=1 \\ \zeta \neq 1}} \frac{1}{q \zeta-1} \frac{1}{\zeta^{-h}-1} H_{n}\left((q \zeta)^{-1} ; q\right) .
$$

Note that by (6) we have

$$
\lim _{a \rightarrow 1}\left\{s_{n ; q}(h, k)-\frac{\alpha_{n} B_{1}}{[k]^{n} k}\right\}=\frac{1}{n+1}\left\{s_{n+1}(h, k)-\frac{B_{1} B_{n+1}}{k^{n+1}}\right\},
$$

where $s_{n+1}(h, k)$ are classical $n+1$-th higher-order Dedekind sums introduced by Apostol [1]. In the next section we prove the reciprocity law for these $q$-Dedekind sums.

\section{1-2. Reciprocity law for $s_{n ; q}(h, k)$.}

Our main result in this section is described as follows:

THEOREM 1. For any non-negative integer $n$ and positive integers $h$ and $k$ such that $(h, k)=1$, we have

$$
\begin{aligned}
{[k]^{n} s_{n ; q}(h, k) } & +[h]^{n} s_{n ; q}(k, h) \\
& =\left\{(q-1) \alpha\left(q^{k}\right) \alpha\left(q^{h}\right)[k][h]+\alpha\left(q^{k}\right)[k]+\alpha\left(q^{h}\right)[h]\right\}^{n} \\
& -\frac{1}{k h}\left\{(q-1) \alpha^{2}(q)+\alpha(q)+\alpha(q)\right\}^{n}-\frac{1}{k h} \alpha_{n}(q),
\end{aligned}
$$

with the usual convention about replacing $\alpha^{i}(q)$ by $\alpha_{i}(q)$ (note that $\alpha^{0}(q)$ is equal to $a_{0}(q)$ not necessarily to 1$)$.

Our result implies Apostol's reciprocity law [1, Theorem 1]. In order to prove Theorem 1, we need several lemmas. 
Let $f(t)$ and $g(t)$ be power series which have the following representations:

$$
f(t)=\sum_{n=0}^{\infty} f_{n} e^{[n] t}=\sum_{n=0}^{\infty} F_{n} \frac{t^{n}}{n !}
$$

and

$$
g(t)=\sum_{n=0}^{\infty} g_{n} e^{[n] t}=\sum_{n=0}^{\infty} G_{n} \frac{t^{n}}{n !}
$$

respectively. Then we have

and

$$
F_{n}=\sum_{m=0}^{\infty} f_{m}[m]^{n}
$$

$$
G_{n}=\sum_{m=0}^{\infty} g_{m}[m]^{n}
$$

for $n \geq 0$ (according to necessity, we assume the convergency of the above series). And we define a $*$ product between $e^{[m] t}$ and $e^{[n] t}$ as follows:

$$
e^{[m] t} * e^{[n] t}=e^{[m+n] t},
$$

and extend it between $f(t)$ and $g(t)$ linearly. Then we have

and the coefficients of $\frac{t^{k}}{k !}$ in (7) are given by

$$
f(t) * g(t)=\sum_{n=0}^{\infty}\left(\sum_{m=0}^{\infty} f_{m} g_{n-m}\right) e^{[n] t},
$$

$$
\begin{aligned}
\sum_{n=0}^{\infty} \sum_{m=0}^{\infty} f_{m} g_{n-m}[n]^{k} & =\sum_{m=0}^{\infty} \sum_{n=0}^{\infty} f_{m} g_{n}[m+n]^{k} \\
& =\sum_{m=0}^{\infty} \sum_{n=0}^{\infty} f_{m} g_{n}\left(q^{m}[n]+[m]\right)^{k} \\
& =\sum_{m=0}^{\infty} \sum_{n=0}^{\infty} f_{m} g_{n} \sum_{i=0}^{k}\left(\begin{array}{c}
k \\
i
\end{array}\right) q^{m i}[n]^{i}[m]^{k-i} \\
& =\sum_{i=0}^{k}\left(\begin{array}{c}
k \\
i
\end{array}\right) G_{i} \sum_{m=0}^{\infty} f_{m} q^{m i}[m]^{k-i} \\
& =\sum_{i=0}^{k}\left(\begin{array}{l}
k \\
i
\end{array}\right) G_{i} \sum_{m=0}^{\infty} f_{m} \sum_{j=0}^{i}\left(\begin{array}{l}
i \\
j
\end{array}\right)(q-1)^{j}[m]^{k-i+j} \\
& =\sum_{i=0}^{k}\left(\begin{array}{l}
i \\
j
\end{array}\right) G_{i} \sum_{j=0}^{i}\left(\begin{array}{l}
i \\
j
\end{array}\right)(q-1)^{j} F_{k-i+j} \\
& =\{(q-1) F G+F+G\}^{k} .
\end{aligned}
$$

Hence we obtain the following:

LEMMA 4. For the above $f(t)$ and $g(t)$, we have

$$
f(t) * g(t)=\sum_{n=0}^{\infty}\{(q-1) F G+F+G\}^{n} \frac{t^{n}}{n !} .
$$


Similarly to [6, Lemma 1], we can express $F_{u ; q}(t)$ by

$$
F_{u ; q}(t)=\left(1-\frac{1}{u}\right) \sum_{n=0}^{\infty} u^{-n} e^{[n] t}
$$

if $|u|>1$. Hence the $*$ product is defined between $F_{u ; q}(t)$ and $F_{v ; q}(t)$ if $|u|>1$ and $|u|>1$, and the following holds:

Lemma 5. For any distinct complex numbers $u$ and $v$ such that $|u|>1$ and $|v|>1$, we have

$$
F_{u ; q}(t) * F_{v ; q}(t)=\frac{1-v}{u-v} F_{u ; q}(t)+\frac{1-u}{v-u} F_{v ; q}(t) .
$$

Proof. Using the $*$ product, the defining equation $[6,(6)]$ of $F_{u ; q}(t)$ is expressed by

$$
\frac{1}{u} e^{t} * F_{u ; q}(t)=F_{u ; q}(t)+\frac{1}{u}-1,
$$

where we regard $e^{t}$ as $e^{[1] t}$. Hence we obtain

$$
\begin{aligned}
\left(\frac{1}{u} e^{t} * F_{u ; q}(t)\right) *\left(\frac{1}{v} e^{t} * F_{v ; q}(t)\right) & =F_{u ; q}(t) * F_{v ; q}(t)+\left(\frac{1}{v}-1\right) F_{u ; q}(t) \\
& +\left(\frac{1}{u}-1\right) F_{v ; q}(t)+\left(\frac{1}{u}-1\right)\left(\frac{1}{v}-1\right) .
\end{aligned}
$$

And the left hand side of the above equality is equal to

$$
\frac{1}{u v} e^{[2] t} *\left(F_{u ; q}(t) * F_{v ; q}(t)\right)=\frac{1}{u v} e^{[2] t}\left(F_{u ; q} * F_{v ; q}\right)\left(q^{2} t\right) .
$$

Therefore $F_{u ; q}(t) * F_{v ; q}(t)$ is a solution of

$$
\frac{1}{u v} e^{[2] t} f\left(q^{2} t\right)=f(t)+\left(\frac{1}{v}-1\right) F_{u ; q}(t)+\left(\frac{1}{u}-1\right) F_{v ; q}(t)+\left(\frac{1}{u}-1\right)\left(\frac{1}{v}-1\right),
$$

and the uniqueness is showed easily. On the other hand, we can see by a short calculation that the right hand side of (9) satisfies (10). This completes the proof of Lemma 5.

Since the $*$ product is defined between $A_{q^{k}}([k] t)$ and $A_{q^{k}}([h] t)$, we obtain the following by Lemma 5 :

LEMma 6. For any positive integers $h$ and $k$ such that $(h, k)=1$, we have

$$
A_{q^{k}}([k] t) * A_{q^{h}}([h] t)=\frac{1}{k} \sum_{\substack{\zeta k=1 \\ \zeta \neq 1}} \frac{1}{q \zeta-1} \frac{1}{\zeta^{-h}-1} F_{(q \zeta)^{-1 ; q}}(t)
$$




$$
\begin{aligned}
& +\frac{1}{h} \sum_{\substack{\eta^{h}=1 \\
\eta \neq 1}} \frac{1}{q \eta-1} \frac{1}{\eta^{-k}-1} F_{(q \eta)-1 ; q}(t) \\
& +\frac{1}{k h} A_{q}(t) * A_{q}(t)-\frac{k+h-2}{2 k h} A_{q}(t) .
\end{aligned}
$$

Proof. Noting

$$
\sum_{\substack{\zeta=1 \\ \zeta \neq 1}} \frac{1}{\zeta-1}=\frac{1-k}{2},
$$

we can see easily by Lemma 5 .

By comparing the coefficients of $\frac{t^{n}}{n !}$ in (11) and using Lemma 4, we obtain the following:

LEMMA 7. For any non-negative integer $n$ and positive integers $h$ and $k$ such that $(h, k)=1$, we have

$$
\begin{aligned}
\left\{(q-1) \alpha\left(q^{k}\right) \alpha\right. & \left.\left(q^{h}\right)[k][h]+\alpha\left(q^{k}\right)[k]+a\left(q^{h}\right)[h]\right\}^{n} \\
& =\frac{1}{k} \sum_{\substack{\zeta^{k}=1 \\
\zeta \neq 1}} \frac{1}{q \zeta-1} \frac{1}{\zeta^{-h}-1} H_{n}\left((q \zeta)^{-1} ; q\right) \\
& +\frac{1}{h} \sum_{\substack{\eta^{h}=1 \\
\eta \neq 1}} \frac{1}{q \eta-1} \frac{1}{\eta^{-k}-1} H_{n}\left((q \eta)^{-1} ; q\right) \\
& +\frac{1}{k h}\left\{(q-1) \alpha^{2}(q)+\alpha(q)+\alpha(q)\right\}^{n}-\frac{k+h-2}{2 k h} \alpha_{n}(q) .
\end{aligned}
$$

At present position, we can see the reciprocity low for $q$-Dedekind sums immediately from Lemmas 3 and 7 . We done the proof of Theorem 1.

Finally we conclude this part by raising the following questions:

(i) Find the reciprocity law for generalized $q$-Dedekind sums

$$
\sum_{a \bmod k} \bar{\alpha}_{m}\left(\frac{a}{k} ; q^{k}\right) \bar{\alpha}_{n}\left(\frac{a h}{k} ; q^{k}\right) .
$$

(ii) Determine the relationship between $q$-Dedekind sums and Lambert series.

\section{Generalization of construction of $q$-analogue}

In this part, we construct $q$-analogue of formal power series which satisfy some conditions (for example $F_{u ; 1}(t)$ and $G_{1}(t)$ etc.). The $*$ product introduced in the first part is defined between power series in $e^{t}$, so in order to treat $G_{1}(t)$, we need to extend it to polynomials in $t$ at least.

For fixed complex number $q$ such that $|q|<1$, we define an operator $\varphi_{q}$ on 
formal power series ring $\mathbf{C}[[t]]$ as follows :

Definition 4. For $f(t) \in \mathbf{C}[[t]]$, we define

$$
\varphi_{q}(f(t))=f(t)+(q-1) \frac{d}{d t} f(t) .
$$

We construct $q$-analogue based on the operator $\varphi_{q}$. And the following Lemma is essential in our theory:

Lemma 8. There uniquely exists a $\mathbf{C}$-multilinear map $\Phi_{n}$ for each positive integer $n$ :

$$
\Phi_{n}: \underbrace{\mathbf{C}[[t]] \times \cdots \times \mathbf{C}[[t]]}_{n \text { times }} \longrightarrow \mathbf{C}[[t]]
$$

such that

(i) $\Phi_{n}\left(f_{1}, \ldots, f_{n}\right) \equiv f_{1}(0) \cdots f_{n}(0) \bmod \operatorname{deg} 1$,

(ii) $\Phi_{n}\left(f_{1}, \ldots, f_{n-1}, 1\right)=\Phi_{n-1}\left(f_{1}, \ldots, f_{n-1}\right)$ if $n>1$,

(iii) $\varphi_{q}\left(\Phi_{n}\left(f_{1}, \ldots, f_{n}\right)\right)=\Phi_{n}\left(\varphi_{q}\left(f_{1}\right), \ldots, \varphi_{q}\left(f_{n}\right)\right)$,

for $f_{1}, \ldots, f_{n} \in \mathbf{C}[[t]]$.

Proof. The uniqueness of $\Phi_{n}$ for each $n \geq 1$ is showed without difficulty, because $\Phi_{n}\left(t^{m_{1}}, \ldots, t^{m n}\right)$ for non-negative integers $m_{i}(1 \leq i \leq n)$ is uniquely determined by the mathematical induction on $n$ and $m_{1}+\cdots+m_{n}$.

Next we show the existence. For that, we introduce the following bracket $[*, *]=[*, *]_{q}$ from $\mathbf{C}[[t]] \times \mathbf{C}[[t]]$ to $\mathbf{C}[[t]]$ by

$$
[f, g]=\sum_{n=0}^{\infty} f^{(n)} g^{(n)} \frac{(q-1)^{n}}{n !} t^{n},
$$

where $f^{(n)}$ and $g^{(n)}$ mean the $n$-th formal differential of $f$ and $g \in \mathbf{C}[[t]]$, respectively.

Now $1_{\text {id }}$ and $[*, *$ ] satisfy the conditions (i), (ii) and (iii) of Lemma 8 for the cases of $n=1$ and $n=2$, respectively, thus by the uniqueness we have the following:

$$
\Phi_{1}=1_{\mathrm{id}} \text { and } \Phi_{2}=[*, *] .
$$

On the other hand for $n \geq 3$

$$
\underbrace{[\ldots[*, *], *, \ldots, *]}_{n \text { times }}
$$


satisfies the conditions (i), (ii) and (iii) of Lemma 8, so it coincides with $\Phi_{n}$ by the uniqueness. This completes the proof of Lemma 8 .

Hereafter we denote $[f, g]_{q}$ by $f *_{q} g$ for $f$ and $g \in \mathbf{C}[[t]]$, i.e.,

Definition 5.

$$
f *_{q}=g \sum_{n=0}^{\infty} f^{(n)} g^{(n)} \frac{(q-1)^{\mathrm{n}}}{n !} t^{n}
$$

By Lemma 8 , we can investigate the properties of $*_{q}$.

Lemma 9. $\quad *_{q}$ satisfies commutative law, i.e.,

$$
f *_{q} g=g *_{q} f \text {, }
$$

for any $f$ and $g \in \mathbf{C}[[t]]$.

Proof. It is clear because of the defintion of $*_{q}$ or the uniqueness of $\Phi_{2}$.

Lemma $10 . \quad *_{q}$ satisfies associative law, i.e.,

$$
\left(f *_{q} g\right) *_{q} h=f *_{q}\left(g *_{q} h\right),
$$

for any $f, g$ and $h \in \mathbf{C}[[t]]$.

Proof. By the uniqueness of $\Phi_{3}$, both of $[[*, *], *]$ and $[*,[*, *]]$ coincide with $\Phi_{3}$.

Note that $\mathbf{C}[[t]]$ has a product structure with respect to $*_{q}$, by Lemmas 9 and 10 .

Next we explain that $*_{q}$ satisfies a kind of exponential law.

LEMMA 11. For each complex number a, there uniquely exists $f_{a}(t) \in \mathbf{C}[[t]]$, such that

$$
f_{a}(0)=1 \text { and } \varphi_{q}\left(\left(f_{a}(t)\right)=q^{a} f_{a}(t)\right.
$$

Proof. It is obvious that $f_{a}(t)=e^{[a] t}$.

Now we can easily describe that $*_{q}$ satisfies a kind of exponential law as follows:

Proposition 1. For any complex numbers $a$ and $b$, we have 


$$
\Phi_{2}\left(f_{a}, f_{b}\right)=f_{a+b}, \text { i.e., } \quad e^{[a] t} *_{q} e^{[b] t}=e^{[a+b] t}
$$

Proof. By Lemma $8, \Phi_{2}\left(f_{a}, f_{b}\right)$ satisfies the conditions of Lemma 11 for $a+b$, so it coincides with $f_{a+b}$.

Therefore the $*_{q}$ product is regarded as an extension of the $*$ product which is introduced in the first part. And by the above argument, we can construct $q$-analogue of formal power series which satisfy some conditions.

Definition 6. We set the following:

$$
\begin{aligned}
& R=(R,+, \cdot)=\left\{\sum_{n=0}^{\infty} a_{n} e^{n t} \mid \sum_{n=0}^{\infty} a_{n} q^{n} \text { is absolutely convergent. }\right\}, \\
& R_{q}=\left(R_{q},+, *_{q}\right)=\left\{\sum_{n=0}^{\infty} a_{n} q^{n} e^{[n] t} \mid \sum_{n=0}^{\infty} a_{n} q^{n} \text { is absolutely convergent. }\right\}, \\
& R_{q}[t]_{q}=\left(R_{q}[t]_{q},+, *_{q}\right)=\{\sum_{n=0}^{\text {finite }} f_{n} *_{q} \underbrace{t *_{q} \cdots *_{q} t}_{n \text { times }} \mid f_{n} \in R_{q} \text { for each } n\} .
\end{aligned}
$$

Note that all of elements of $R_{q}[t]_{q}$ can be viewed as those of $\mathbf{C}[[t]]$ because of the condition on $R$, so let $i_{q}$ be the inclusion map, i.e.,

$$
R_{q}[t]_{q} \stackrel{i q}{\hookrightarrow} \mathbf{C}[[t]] .
$$

Definition 7. For each $f=\sum_{n=0}^{\infty} a_{n} e^{n t} \in R$, we define

$$
f^{* q}=\sum_{n=0}^{\infty} a_{n} e^{[n] t}
$$

And we define $\phi_{q}$ as follows:

$$
\begin{aligned}
\phi_{q}: R[t] & \longrightarrow R_{q}[t]_{q} \\
\sum_{n=0}^{\text {finite }} f_{n} t^{n} & \mapsto \sum_{n=0}^{\text {finite }} f_{n}^{* q} *_{q} \underbrace{t *_{q} \cdots *_{q} t .}_{n \text { times }}
\end{aligned}
$$

If we extend $\varphi_{q}$ to $R_{q}[t]_{q}$, then it is a ring endomorphism on $R_{q}[t]_{q}$ with respect to + and $*_{q}$, and the following diagram commutes:

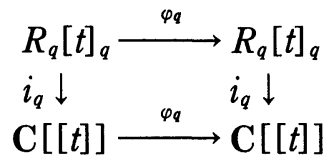

And by the definitions, it is clear that $i_{q} \circ \varphi_{q} \circ \psi_{q}$ is $\mathbf{C}$-homomorphism from $R[t]$ 
to $\mathbf{C}[[t]]$. So we define $q$-analogue for each element of $R[t]$ as follows:

Definition 8. For each $f \in R[t]$, we define a $q$-analogue $f_{q}$ of $f$ by

$$
f_{q}=i_{q} \circ \varphi_{q} \circ \psi_{q}(f) \text {. }
$$

EXAmples. Using our construction of $q$-analogue, the generating functions of Carlitz's $q$-Bernoulli numbers and $q$-Euler numbers are expressed as follows:

$$
\begin{aligned}
& G_{q}(t)=\left(G_{1}(t)\right)_{q}, \\
& \frac{u}{u-1} F_{u ; q}(t)=\left(\frac{u q}{u q-1} F_{u q ; 1}(t)\right)_{q} \text { and } \\
& A_{q}(t)=\left(\frac{G_{1}(t)}{t}\right)_{q} .
\end{aligned}
$$

Since $i_{q} \circ \varphi_{q} \circ \phi_{q}$ is $\mathbf{C}$-homomorphis, we obtain the following:

THEOREM 2. For $H\left(t_{1}, \ldots, t_{n}\right)=\sum_{m_{1}, \ldots, m_{n} \in \mathbf{C}} a_{m_{1}, \ldots, m_{n}} t_{1}^{m_{1}} \cdots t_{n}^{m_{n}} \in \mathbf{C}\left[t_{1}, \ldots, t_{n}\right]$, we formally set

$$
H^{*_{q}}\left(t_{1}, \ldots, t_{n}\right)=\sum_{m_{1}, \ldots, m_{n} \in \mathbf{C}} a_{m_{1}, \ldots, m_{n}} t_{1} \underbrace{*_{q} \cdots *_{q} t_{1}}_{m_{1} \text { trmes }} *_{q} \cdots *_{q} \underbrace{t_{n} *_{q} \cdots *_{q} t_{n} .}_{m_{n} \text { times }}
$$

And for $f_{1}, \ldots, f_{n} \in R[t]$, if the following holds

$$
H\left(f_{1}, \ldots, f_{n}\right)=0,
$$

then the following holds

$$
H^{*_{q}}\left(\left(f_{1}\right)_{q}, \ldots,\left(f_{n}\right)_{q}\right)=0
$$

EXAMPLES. The motivation of this paper is the construction of $q$-analogue of (1) (see Introduction). At present stage, it is clear, i.e., we have the following by Theorem 2:

$$
F_{u ; q}(t) *_{q} F_{v ; q}(t)=\frac{1-v}{u-v} F_{u ; q}(t)+\frac{1-u}{v-u} F_{v ; q}(t),
$$

for any distinct complex numbers $u$ and $v$ such that $|u|>1$ and $|v|>1$.

Next we shall look back the argument of the first part through our theory. For that, we need two Lemmas.

Lemma 12. For any $f(t)=g(t) h(t)$ such that $g(t)$ is monomial in $t$ and $h(t) \in R$, and positive integer $k$, we have 


$$
(f(k t))_{q}=g\left(\frac{k}{[k]}\right) f_{q k}([k] t)
$$

Proof. By the definition and a short calculation, we can obtain what we want.

Lemma 13. For any $f(t) \in \mathbf{C}[[t]]$ and $x \in \mathbf{C}$, we have

$$
f(t) *_{q} e^{[x] t}=f\left(q^{x} t\right) e^{[x] t} .
$$

Proof. This is also clear, by the definition and a short calculation.

Now since classical Dedekind sums $s_{n}(h, k)$ are expresses as follows [3, (6.5)]:

$$
s_{n}(h, k)=\frac{B_{1} B_{n}}{k^{n}}+\frac{n}{k^{n}} \sum_{\substack{\zeta k=1 \\ \zeta \neq 1}} \frac{1}{\zeta-1} \frac{1}{\zeta^{-h}-1} H_{n-1}\left(\zeta^{-1} ; 1\right)
$$

for $n \geq 1$ and $s_{0}(h, k)=B_{1}$, their generating function $S_{h, k}(t)$ is given by

$$
B_{1} G_{1}\left(\frac{t}{k}\right)+\frac{t}{k} \sum_{\substack{\zeta k=1 \\ \zeta \neq 1}} \frac{1}{\zeta-1} \frac{1}{\zeta^{-h}-1} F_{\zeta^{-1}}\left(\frac{t}{k}\right) \text {. }
$$

Hence we have

$$
S_{h, k}(k t)=B_{1} G_{1}(t)+t \sum_{\substack{\zeta k=1 \\ \zeta \neq 1}} \frac{1}{\zeta-1} \frac{1}{\zeta^{-h}-1} F_{\zeta^{-1}}(t) .
$$

Directly we can construct $q$-analogue of the above equality, but by treating $\frac{S_{h, k}(k t)}{k t}$, we can obtain a simpler result. By Theorem 2, we have

$$
\left(\frac{S_{h, k}(k t)}{k t}\right)_{q}=\frac{B_{1}}{k} A_{q}(t)+\frac{1}{k} \sum_{\substack{\zeta k=1 \\ \zeta \neq 1}} \frac{1}{q \zeta-1} \frac{1}{\zeta^{-h}-1} F_{(q \zeta)-1 ; q}(t) .
$$

Therefore, by Lemmas 3 and 12, we can express our $q$-Dedekind sums which were defined in the first part as the coefficients of $\left(\frac{S_{h, k}(t)}{t}\right)_{q}$. And we can deduce Lemma 6, which implies the reciprocity law for $q$-Dedekind sums from

$$
\begin{aligned}
\frac{G_{1}(k t)}{k t} \frac{G_{1}(h t)}{h t}= & \frac{1}{k} \sum_{\substack{\zeta k=1 \\
\zeta \neq 1}} \frac{1}{\zeta-1} \frac{1}{\zeta^{-h}-1} F_{\zeta^{-1 ; 1}}(t) \\
& +\frac{1}{h} \sum_{\substack{\eta^{h}=1 \\
\eta \neq 1}} \frac{1}{\eta-1} \frac{1}{\eta^{-k}-1} F_{\eta^{-1} ; 1}(t) \\
& +\frac{1}{k h} \frac{G_{1}(t)}{t} \frac{G_{1}(t)}{t}-\frac{k+h-2}{2 k h} \frac{G_{1}(t)}{t}
\end{aligned}
$$


which is equivalent to $[4,(3.4)]$.

Finally we conclude this paper by raising two examples about $q$-Bernoulli polynomials and $q$-Euler polynomials. At first, we treat $q$-Bernoulli poloynomials. For a prime number $p$, the following equation plays an important role in constructing the $p$-adic Bernoulli measure on $\mathbf{Z}_{p}$ :

$$
k^{n-1} \sum_{a=0}^{k-1} B_{n}\left(\frac{x+a}{k}\right)=B_{n}(x),
$$

for any positive integer $k$ and non-negative integer $n$. $q$-Analogue of (12) is given by Carlitz [2, (5.9)] and that of the equation for Euler polynomials is given by the author [6, Lemma 2]. Using our construction of $q$-analogue, we can clearly explain them as follows:

Now $q$-analogue of (12) for $n \geq 0$ is expressed by

$$
[k]^{n-1} \sum_{a=0}^{k-1} q^{a} \beta_{n}\left(\frac{x+a}{k} ; q^{a}\right)=\beta_{n}(x: q) .
$$

Since the generating function of $q$-Bernoulli polynomials is given by

$$
\sum_{n=0}^{\infty} \beta_{n}(x ; q) \frac{t^{n}}{n !}=G_{q}\left(q^{x} t\right) e^{[x] t},
$$

(13) is equivalent to the following:

$$
G_{q}(t)=\frac{1}{[k]} \sum_{a=0}^{k-1} q^{a} G_{q^{k}}\left(q^{a}[k] t\right) e^{[a] t} .
$$

On the other hand, the classical equation (i.e., in the case of $q=1$ ) which corresponds to the above equation is

$$
G_{1}(t)=\frac{1}{k} \sum_{a=0}^{k-1} G_{1}(k t) e^{a t},
$$

and it is trivial. So we can deduce (14) from (15) by Theorem 2, Lemmas 12 and 13.

Next we explain an example about $q$-Euler polynomials [6, Lemma 2], which was already used in the proof of Lemma 1 :

$$
[k]^{n} \sum_{a=0}^{k-1} \frac{u^{-a}}{u^{-k}-1} H_{n}\left(\frac{x+a}{k}, u^{k} ; q^{k}\right)=\frac{1}{u^{-1}-1} H_{n}(x, u ; q),
$$

for $n \geq 0$. Similarly to the above argument, we can deduce (16) from a trivial

$$
\frac{1}{u^{-1}-1} F_{u ; 1}(t)=\sum_{a=0}^{k-1} \frac{u^{-a}}{u^{-k}-1} F_{u^{k} ; 1}(k t) e^{a t} .
$$




\section{REFERENCES}

[1] T. M. Apostol, Generalized Dedekind sums and transformation formulae of certain Lambert series, Duke Math. J., 17 (1950), 147-157.

[2] L. Carlitz, $q$-Bernoulli numbers and polynomials, Duke Math. J., 15 (1948), $987-1000$.

[ 3 ] - Some theorems on generalized Dedekind sums, Pacific J. Math., 3 (1953), $513-522$.

[ 4 ] The reciproctiy theorem for Dedekind sums, Pacific J. Math., 3(1953), 523-527.

[ 5 ] - $q$-Bernoulli and Eulerian numbers, Trans. Amer. Soc., 76 (1954), 332-350.

[6] J. Satoh, $q$-Analogue of Riemann's $\zeta$-function and $q$-Euler numbers, J. Nunmber Theory, 31 (1989), 346-362.

Konan Women's Junior College

Takaya-cho 172, Konan 483, Japan 